\title{
Entropy and enthalpy for triggering cutaneous erythema
}

\author{
Steven L. Jacques, ${ }^{*}$ and Joseph T. Walsh ${ }^{* \dagger}$ \\ *Wellman Laboratories, Massachusetts General Hospital \\ Boston, MA, USA \\ ${ }^{\dagger}$ Harvard Medical School-M.I.T., Cambridge, MA, USA \\ *jacquess@ohsu.edu
}

Received 3 September 2014

Accepted 15 December 2014

Published 28 January 2015

\begin{abstract}
The molar entropy $(\Delta S)$ and molar enthalpy $(\Delta H)$ for the denaturation event that triggers cutaneous erythema was determined to be $\Delta S=190 \mathrm{~J} /($ mole K $)$ and $\Delta H=146.9 \times 10^{3} \mathrm{~J} / \mathrm{mole}$. The experiment involved placing heated water against the skin of the forearm, for a range of temperatures and exposure times. Exposing the skin to $45^{\circ} \mathrm{C}$ for $22 \mathrm{~s}$ was at the threshold for producing erythema, which was similar to the threshold for a slight degree of pain.
\end{abstract}

Keywords: Skin; erythema; thermal; entropy; enthalpy.

\section{Introduction}

This paper is included in the special issue honoring Valery Tuchin, who has greatly contributed to the field of biophotonics and biomedical optics. Professor Tuchin has reported on various aspects of tissue type and structure that affect the appearance and optical behavior of tissues. Blood is a strong and dominant optical absorber in the visible and near infrared spectrum. Vascular responsivity is a key biological parameter characterizing the state of a tissue. The responsivity of blood perfusion to heat is observed as a dilation of vessels. Skin is a tissue that allows easy observation of such vascular dilation, called erythema. Therefore, understanding the temperature history that elicits cutaneous erythema would allow one to recognize when the skin's responsivity departs from expected behavior. Such a report is appropriate for a special issue honoring Professor Tuchin.

This paper reports on the temperature history that elicits cutaneous erythema, which occurs when the superficial vasculature of the skin dilates in response to thermal exposure. In 1984, we conducted an experiment (never published) to document the threshold thermal history that caused cutaneous erythema.

\$Corresponding author.

\$Current address: Oregon Health \& Science University, 3303 SW Bond Ave., Portland, OR, USA 97239.

This is an Open Access article published by World Scientific Publishing Company. It is distributed under the terms of the Creative Commons Attribution 3.0 (CC-BY) License. Further distribution of this work is permitted, provided the original work is properly cited. 


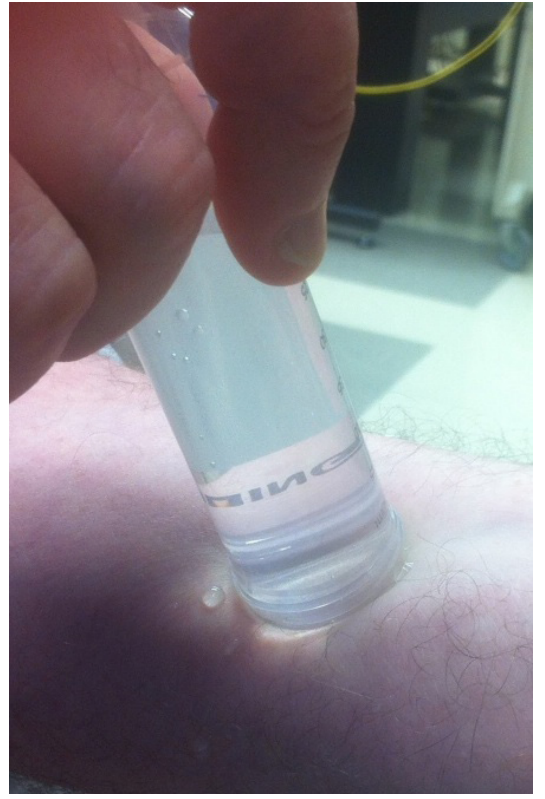

Fig. 1. Photo of 50-mL centrifuge tube filled with heated water and placed on forearm skin.

\section{Experimental Method}

A 50-mL plastic centrifuge tube was filled with water, heated to a specific temperature and measured by thermometer. The tube was inverted against the skin of the forearm and held for a specific time duration, then removed and the skin site blotted with a towel. The site was observed by eye for $20 \mathrm{~s}$, and any sign of visible erythema was scored as a 1. Lack of erythema was scored as a 0. The process was repeated for various temperatures and various time durations. While higher temperatures/ times caused stronger erythema than lower temperatures/times, the onset or lack of onset of erythema was quite distinct, and this experiment documented the threshold for erythema. Figure 1 shows a centrifuge tube placed against the skin. Experiments were conducted on the forearm of SLJ.

\section{Results}

Figure 2 shows the data plotted as exposure time $[s]$ versus exposure temperature $\left[{ }^{\circ} \mathrm{C}\right]$, red circles indicating erythema, black crosses indicating no erythema.

The data were analyzed by a fitting routine in which a range of $\Delta H$ and $\Delta S$ values were assumed and a threshold curve for erythema onset was calculated (see next paragraph). The observed data was

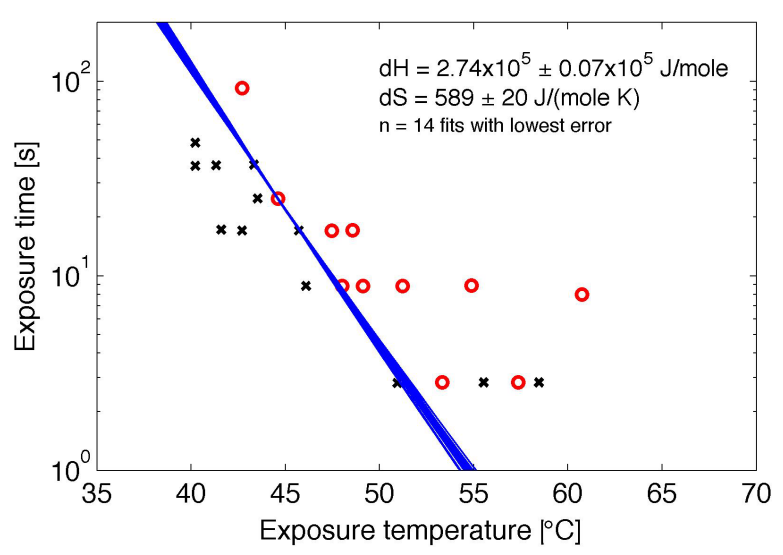

Fig. 2. Experimental observation of temperature-history induced erythema on forearm skin. Holding a 50-mL-volume tube filled with heated water against the skin for a given time either caused no observable erythema (black crosses) or elicited an observable erythema (red circles). The data were fit by Eq. (1) to yield the $\Delta H$ and $\Delta S$ that minimized the number of FPs (2) and FNs (3), denoted by thin vertical red lines.

scored as true positive (TP), false positive (FP), true negative $(\mathrm{TN})$ and false negative $(\mathrm{FN})$ relative to this threshold curve. The number of data in error $\left(N_{\text {error }}=\mathrm{FP}+\mathrm{FN}\right)$ relative to the threshold curve was recorded for each $\Delta H, \Delta S$ pair. Figure 3(a) shows the map of $N_{\text {error }}$ versus the range of $\Delta H$ and $\Delta S$ values, indicating a line of minimum error extending across the map. This line behaved as $\Delta S=-272.23+3.1456 \times 10^{3} \Delta H$. Figure $3(\mathrm{~b})$ shows the value of $N_{\text {error }}$ versus $\Delta H$ along this line of minima, indicating that a unique minimum occurred at $\Delta H=2.74 \times 10^{5} \pm 0.07 \times 10^{5} \mathrm{~J} / \mathrm{mole}, \quad \Delta S=$ $589 \pm 20 \mathrm{~J} /($ mole $\mathrm{K}), n=14 \Delta H, \Delta S$ pairs, which allowed $N_{\text {error }}=2$ (the two FPs are at $55.5^{\circ} \mathrm{C}$ and $\left.58.5^{\circ} \mathrm{C}\right)$. The value of $\Delta S$ cited here is calculated $\Delta S=-272.23+3.1456 \times 10^{3}($ mean $\Delta H)$.

The method for creating the threshold curve for erythema onset is now described. The choice of $\Delta H, \Delta S$ specifies a rate of denaturation $\left(k\left[\mathrm{~s}^{-1}\right]\right)$ such that the transition from a native to denatured state behaves as a time-dependent loss of native state:

$$
N(t)=N_{0} \mathrm{e}^{-k t}
$$

where $N_{0}$ is the initial number of native species, $t$ is the exposure time, and $N(t)$ describes the exponential decay of native species toward complete denaturation. The rate constant $k$ is related to $\Delta S$ and $\Delta H$ by the expression:

$$
k=\frac{k_{B} T}{h} \mathrm{e}^{\Delta S / R} \mathrm{e}^{-\Delta H /(R T)},
$$




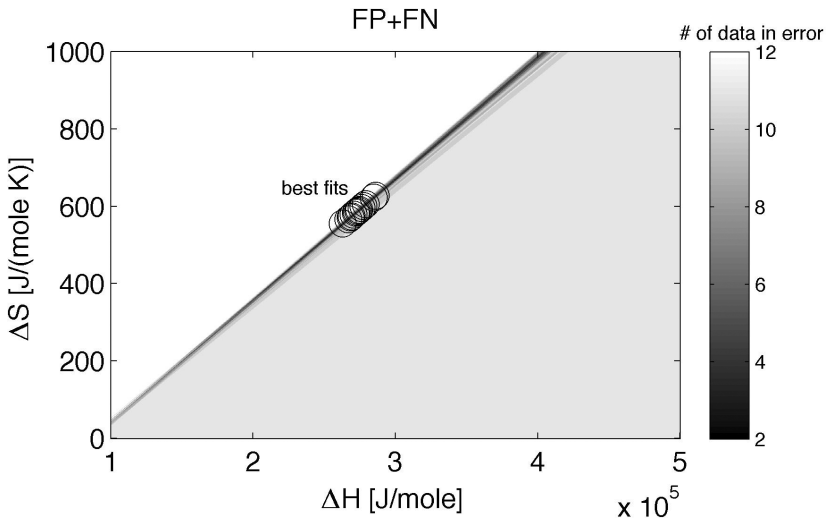

(a)

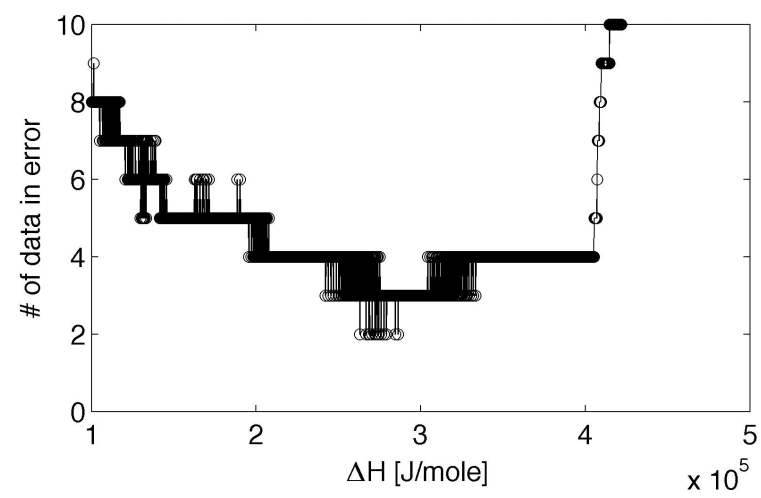

(b)

Fig. 3. Fitting the data for $\Delta H[\mathrm{~J} /$ mole $], \Delta S[\mathrm{~J} /($ mole $\mathrm{K})]$ that elicits erythema. (a) Mapping the number of data in error $\left(N_{\text {error }}=\mathrm{FP}+\mathrm{FN}\right)$ versus a broad range of $\Delta H, \Delta S$ pairs. There is a locus of minima in $N_{\text {error }}$ along a line over the full range of $\Delta H$, but the values of $N_{\text {error }}$ are minimal (equal to 2) at a central region. (b) Shows the error values for the range of $\Delta H, \Delta S$ pairs tested. The figure plots the value of $N_{\text {error }}$ versus $\Delta H$ for all possible $\Delta S$ choices, and indicates a cluster of minima. The mean value of $\Delta H$ for these minima is $2.74 \times 10^{5} \mathrm{~J} /$ mole.

where

$$
\begin{aligned}
k_{B} & =1.38 \times 10^{-23}[\mathrm{~J} / \mathrm{K}] \text { (Boltzmann constant) } \\
h & =6.26 \times 10^{-34}[\mathrm{Js}] \text { (Planck constant) } \\
T & =273.15+T_{\operatorname{deg} C}[\mathrm{~K}] \text { (temperature in Kelvin) } \\
R & =8.314[\mathrm{~J} /(\text { mole K })] \text { (gas constant) } .
\end{aligned}
$$

The damage integral, $\Omega$ [dimensionless], is defined

$$
\Omega(t)=\int_{0}^{t} k\left(T\left(t^{\prime}\right)\right) d t^{\prime}
$$

where the rate constant $k$ varies as the temperature changes over time, $T(t)$. When $\Omega$ reaches 1 , the number of native species has reduced to $0.37 N_{0}$, and by convention this thermal damage level is regarded as the threshold damage yielding the observed endpoint, which in this case is erythema. If the temperature is constant during a particular test, then $\Omega=k t$, and by the convention of $\Omega=1$ for threshold effect, the threshold exposure time is $t=1 / k$. This calculated exposure time plotted versus temperature is the curve in Fig. 1 that constitutes the predicted threshold for triggering erythema.

\section{Discussion}

Although this early experiment collected limited data, the data offer a first approximation to the temperature history that elicits erythema. The experiment deserves to be repeated to provide more data and a better characterization of the $\Delta H$ and $\Delta S$ for the denaturation event that triggers cutaneous erythema. Measurements on a set of subjects could specify the variation in the thermal responsivity between individuals, and studies could address the dependence of responsivitiy on age, gender, race and region of the body. However, it should be cautioned that such experiments are somewhat painful. In the experiments of this paper, it was observed that a temperature of $45^{\circ} \mathrm{C}$ for $22 \mathrm{~s}$ is at the threshold for erythema, but involves a tolerable but definite degree of pain. In general, our observation was that the pain threshold was close to the threshold for erythema. Optically following the temporal development of erythema during exposure to $45^{\circ} \mathrm{C}$ heated water may constitute a measurement of the rate constant $k$ in Eq. (1), which could be a practical assay for vascular responsivity.

An aspect of the experiment that deserves comment is the role that thermal diffusion plays. Upon placing the heated water against the skin, the heat must diffuse into the upper $\sim 250 \mu \mathrm{m}$ of skin where the superficial vasculature is located. Heat diffusion over such a distance requires approximately $t=$ $d^{2} /(4 \alpha)=0.11 \mathrm{~s}$, where $d=0.0250 \mathrm{~cm}, \alpha=1.4 \times$ $10^{-3} \mathrm{~cm}^{2} / \mathrm{s}$ which is the diffusivity of heat in water. So the experiment should not be limited by thermal diffusion until exposure times drop to less than a couple seconds. The heat sink provided by the cutaneous vasculature is a complicating factor. The temperature within the skin is a balance between the topical flux of heat and the removal of heat by the superficial blood perfusion. Hence, the $\Delta S, \Delta H$ values cited in this paper are a descriptive 
characterization since the actual temperature within the site of action for erythema was not exactly known.

Reviewing the literature, we could not find any reports on the threshold temperature history that elicited erythema. There were a number of reports that mentioned erythema after exposures that achieved skin burns, but the data did not contribute to understanding the threshold for onset of erythema.

One report of interest ${ }^{1}$ reported on the threshold for pain when heating the skin with a topical Peltier-regulated thermode at a rate of $0.4^{\circ} \mathrm{C} / \mathrm{s}$, starting at $30^{\circ} \mathrm{C}$. The subjects pushed a button when the sensation of pain was uncomfortable, and the temperature was noted. Two experiments were conducted, yielding threshold temperatures of $40^{\circ} \mathrm{C}$ in the first study and $44^{\circ} \mathrm{C}$ in the second study. Figure 4(a) shows the rise in temperature during the studies, and Fig. 4(b) shows the increase in the damage integral, $\Omega_{\text {erythema }}$, based on the $\Delta H, \Delta S$ values that characterize the onset of erythema. At the onset of pain reported at $44^{\circ} \mathrm{C}$ after $35 \mathrm{~s}$ of heating in the second study, the value of $\Omega_{\text {erythema }}$ reaches 0.26 . At $46.0 \mathrm{~s}$ of heating, the $\Omega_{\text {erythema }}$ reaches 1.0, the threshold for erythema. The discrepancy between $35 \mathrm{~s}$ and $46 \mathrm{~s}(11 \mathrm{~s})$ is small and the temperature histories that elicit pain and erythema are similar. This similarity is consistent with our experimental observation that the threshold for erythema correlated well with the sensation of mild pain.

Hopefully, this report may motivate future experiments on optically assaying vascular responsivity to thermal exposures as a means of recognizing vascular pathology or following modulation of vascular behavior by drugs. Optical measurements

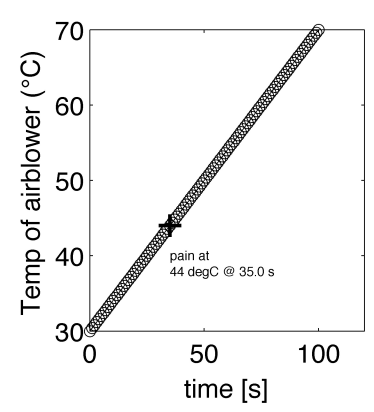

(a)

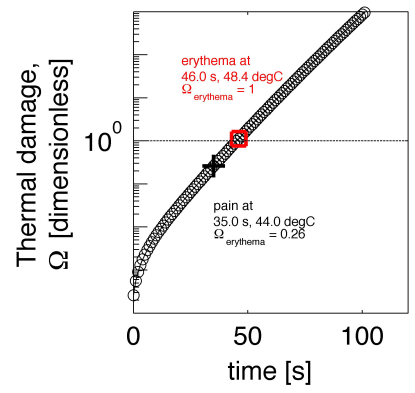

(b)
Fig. 4. Simulating the study by Sycha et al. ${ }^{1}$ on the onset of pain induced by topical cutaneous heating. (a) The temperature rise induced topically on the skin by a Peltier-regulated thermode, starting at $30^{\circ} \mathrm{C}$ and increasing over time at $0.4^{\circ} \mathrm{C} / \mathrm{s}$. Pain reported when temperature reached $44^{\circ} \mathrm{C}$ which occurred at $35 \mathrm{~s}$. (b) The damage integral, $\Omega_{\text {erythema }}$, that elicits erythema, calculated for the temperature history in Fig. 4(a) based on the $\Delta H$ and $\Delta S$ of erythema and calculated by Eqs. (2) and (3). The damage eliciting erythema occurs at $46 \mathrm{~s}$, when the temperature has reached $48.8^{\circ} \mathrm{C}$.

are especially attractive for measuring changes in both larger vessel sizes and the average blood volume fraction of the microvasculature. An optical technique can both heat the skin with light and monitor the vascular response in one placement of a test device. There is opportunity for optical assessment of vascular status and behavior based on the vascular response to a calibrated optical heating.

\section{References}

1. T. Sycha, B. Gustorff, S. Lehr, A. Tanew, H.-G. Eichler, L. Schmetterer, "A simple pain model for the evaluation of analgesic effects of NSAIDs in healthy subjects," Br. J. Clin. Pharmacol. 56, 165172 (2003). 